\title{
Attribute-Based Subsequence Matching and Mining
}

\author{
Yu PENG *, Raymond Chi-Wing Wong *, Liangliang Ye *, Philip S. Yu \# \\ *The Hong Kong University of Science and Technology \#University of Illinois at Chicago \\ \{gracepy, raywong, llye\}@cse.ust.hk \\ psyudcs.uic.edu
}

\begin{abstract}
Sequence analysis is very important in our daily life. Typically, each sequence is associated with an ordered list of elements. For example, in a movie rental application, a customer's movie rental record containing an ordered list of movies is a sequence example. Most studies about sequence analysis focus on subsequence matching which finds all sequences stored in the database such that a given query sequence is a subsequence of each of these sequences. In many applications, elements are associated with properties or attributes. For example, each movie is associated with some attributes like "Director" and "Actors". Unfortunately, to the best of our knowledge, all existing studies about sequence analysis do not consider the attributes of elements.
\end{abstract}

In this paper, we propose two problems. The first problem is: given a query sequence and a set of sequences, considering the attributes of elements, we want to find all sequences which are matched by this query sequence. This problem is called attributebased subsequence matching (ASM). All existing applications for the traditional subsequence matching problem can also be applied to our new problem provided that we are given the attributes of elements. We propose an efficient algorithm for problem ASM. The key idea to the efficiency of this algorithm is to compress each whole sequence with potentially many associated attributes into just a triplet of numbers. By dealing with these very compressed representations, we greatly speed up the attributebased subsequence matching. The second problem is to find all frequent attribute-based subsequence. We also adapt an existing efficient algorithm for this second problem to show we can use the algorithm developed for the first problem. Empirical studies show that our algorithms are scalable in large datasets. In particular, our algorithms run at least an order of magnitude faster than a straightforward method in most cases. This work can stimulate a number of existing data mining problems which are fundamentally based on subsequence matching such as sequence classification, frequent sequence mining, motif detection and sequence matching in bioinformatics.

\section{INTRODUCTION}

Sequences are one of the important data types in our daily life [1]. A sequence is an ordered list of elements where each element is drawn from a given element domain set. For example, in the movie rental application, each movie in the rental store corresponds to an element and a set of all movies corresponds to the element domain set. Each customer rents a list of movies. This (ordered) list corresponds to a sequence. Table I(a) shows a movie rental record table. Each element in the element domain set is associated with a set of properties or attributes. For instance, in the movie rental application, each movie is associated with some attributes like "Release Year", "Director" and "Actors". The properties of some movies are shown in Table I(b). This table is called a property table. In particular, the director of movie "Titanic" is "James Cameron" and one of the actors is "Leonardo DiCaprio".
Sequence analysis has received a lot of interest from not only database and data mining communities but also bioinformatics communities. Database researchers study subsequence matching [2-6] and similarity search [7] while data mining researchers study frequent subsequence mining [8] and sequence prediction [9]. On the other hand, bioinformatics researchers study DNA sequence alignment [10], motif discovery [11, 12] and sequence classification $[13,14]$.

All of the above sequence analysis applications depend on a fundamental operator called subsequence matching. Given two sequences $s$ and $s^{\prime}$, sequence $s$ is said to be a subsequence of $s^{\prime}$ if for any two elements $e$ and $e^{\prime}$ in $s$ where $e$ occurs before $e^{\prime}$ in $s$, both elements $e$ and $e^{\prime}$ occurs in $s^{\prime}$ and $e$ occurs before $e^{\prime}$ in $s^{\prime}$. We also say that $s$ matches $s^{\prime}$ (or $s^{\prime}$ is matched by $s)$. For example, if $s$ is "Titanic, Inception" and $s^{\prime}$ is "Titanic, The Aviator, Inception", then $s$ is a subsequence of $s^{\prime}$ and thus $s$ matches $s^{\prime}$. Subsequence matching is formulated as follows: given a set of sequences and a query sequence $q$, we want to find all sequences such that $q$ is a subsequence of each of these sequences. In our running example, if $q$ is "Titanic, Inception", Alice's sequence in Table I is one of the answers for subsequence matching.

Unfortunately, the traditional subsequence matching problem fails to answer a lot of interesting questions related to the properties of elements. Consider that we want to study how movie "Titanic" creates a star "Leonardo DiCaprio". In order to do this, we want to know how many customers are interested in watching movies acted by "Leonardo DiCaprio" after they watch movie "Titanic". Note that "Leonardo DiCaprio" is not a movie (or more formally not an element in the element domain set) but is a property value of a movie. The traditional subsequence matching problem cannot achieve this goal because the original problem is based on the elements but not the property values. One may adapt the traditional problem and generate all possible queries in form of "Titanic, $x$ " where $x$ is a movie acted by "Leonardo DiCaprio". If there are $M$ movies acted by "Leonardo DiCaprio", then this adapted approach will issue $M$ queries, which is quite inefficient.

Motivated by this, in this paper, we are studying a new problem called attribute-based subsequence matching (ASM). Informally speaking, the problem is described as follows: given a query sequence which contains some elements and some property values, we want to find all sequences which are matched by this sequence query. We will give a formal definition in Section III.

There are a lot of convincing applications for problem ASM. One application is finding a researcher with a certain back- 
TABLE I

AN EXAMPLE SHOWING THE MOVIE RENTAL APPLICATION

\begin{tabular}{|c|c|}
\hline Customer & List of movies \\
\hline Alice & Titanic, The Aviator, Inception \\
\hline Bob & Titanic, The Aviator \\
\hline Clement & The Departed, The Dark Knight \\
\hline$\ldots$ & $\ldots$ \\
\hline
\end{tabular}

(a) Movie rental record table

\begin{tabular}{|c|c|c|c|c|c|}
\hline Movie Name & $\begin{array}{l}\text { Release } \\
\text { Year }\end{array}$ & Director & Actor 1 & Actor 2 & $\ldots$ \\
\hline Titanic & 1997 & James Cameron & Leonardo DiCaprio & Kate Winslet & $\ldots$ \\
\hline The Aviator & 2004 & Martin Scorsese & Leonardo DiCaprio & Cate Blanchett & $\ldots$ \\
\hline The Departed & 2006 & Martin Scorsese & Leonardo DiCaprio & Matt Damon & $\ldots$ \\
\hline The Dark Knight & 2008 & Christopher Nolan & Christian Bale & Heath Ledger & $\ldots$ \\
\hline Avatar & 2009 & James Cameron & Sam Worthington & Zoe Saldana & $\ldots$ \\
\hline Inception & 2010 & Christopher Nolan & Leonardo DiCaprio & Joseph Gordon-Levitt & $\ldots$ \\
\hline$\ldots$ & $\ldots$ & $\ldots$ & $\ldots$ & $\ldots$ & $\ldots$ \\
\hline
\end{tabular}

(b) Movie property table

ground. Suppose that we are given a number of researchers' background where each sequence of a researcher's background is a series of affiliations. Note that affiliations correspond to elements in our context. Each affiliation can be associated with some properties like "Place" and "Private/Public". UCLA is an example of affiliations and it is a public university located in $L A$. Microsoft Research is another example and it is a private company located in Redmond. One interesting query is to "find all researchers who graduate in $L A$ and find jobs in Microsoft Research". Note that LA is a property value and Microsoft Research is an affiliation.

Another application can be finding a purchase pattern in point-of-sale transactions. Each transaction is a sequence of items (e.g., laptop and mouse). Each item can be associated with some categories. For example, laptop is under category "Electronics" and mouse is under category "Computer Accessories". One interesting query is to find all customers who purchased "laptop" and then purchased some "Computer Accessories". Note that "laptop" is an item and "Computer Accessories" is a category value.

The third application can be finding a biological pattern in protein sequences. In protein sequences, 20 amino-acids form an element set. Similarly, each amino acid is associated with some properties like "side chain polarity", "side chain charge" and "hydropathy index". It is interesting to study the biological patterns related to these properties in the bioinformatics literature. One may want to find all protein sequences containing "glutamic acid", a polar amino acid, and then another polar amino acid to study some chemical reactions for generating medicines and drugs.

It is worth mentioning that problem ASM can be used in a lot of problems about sequence mining and problems about bioinformatics. Sequence classification is one example. We should classify sequences according to not only the elements in the sequence but also their property values. Motif detection over sequences is another example. Based on the property values, we can find some motifs that we have not discovered before. In conclusion, if we can solve problem ASM, all existing sequence mining problems can also be extended for discovering meaningful patterns.

In this paper, we will show that a straightforward implementation by simply re-using some existing algorithms runs inefficiently. Motivated by this, we propose an efficient algorithm for the problem based on Chinese Remainder Theorem. The key idea to the efficiency of this algorithm is using some mathematical techniques in Chinese Reminder Theorem to generate concise synopsis of all sequences. Specifically, each whole sequence with associated attribute values is compressed into a triplet of numbers using the theorem. All of the triplets form the concise synopsis. By using the synopsis, we can find all sequences which are matched by the query sequence efficiently. In other words, this compressed representation greatly speeds up the attribute-based subsequence matching.

Note that each number in the triplet for a sequence is a large number so that it can store the information about a sequence. One may think that operations over large numbers are costly. However, one advantage of using large numbers is that we can make use of efficient bitwise operations over only three large numbers. Compared with the approaches not using the large numbers which involves a lot of operations on non-large numbers, manipulating the large numbers is more efficient because it involves fewer operations on large numbers and these bitwise operations are implemented efficiently.

In order to illustrate how ASM can be used in other important data mining problems, we propose a problem which finds all frequent subsequences based on ASM. Besides, we adapt an existing efficient algorithm called SPAM [8].

Our contributions are summarized as follows. Firstly, to the best of our knowledge, we are the first to propose problem ASM which considers the property table. This problem has a lot of convincing applications. This work can stimulate a number of existing data mining problems which are fundamentally based on subsequence matching such as sequence classification, motif detection and sequence matching. Secondly, we propose a novel algorithm for problem ASM based on Chinese Remainder Theorem. Thirdly, we propose a data mining problem to find all frequent subsequences based on ASM to illustrate how ASM can be used in other data mining problems. Fourthly, we conducted some experiments to show the efficiency of our proposed algorithms.

The rest of the paper is organized as follows. Section II summarizes the related work in the literature. Section III gives the problem definition. Section IV introduces some fundamental concepts which are used in our proposed algorithm. Section V gives our proposed algorithm. Section VI formulates the problem of finding frequent subsequence based on ASM and gives our adapted algorithm for this problem. Section VII gives comprehensive experimental results. Section VIII concludes this paper.

\section{RELATED WORK}

Subsequence matching attracted a lot of attention in the database community, the data mining community and the 
bioinformatic community $[2-4,15,16,10]$. Subsequence matching can be classified into two types: accurate matching and approximate matching. Given a query sequence $q$, accurate (subsequence) matching $[15,16,10]$ is to find all sequences such that $q$ is a subsequence of each of these sequences. On the other hand, approximate (subsequence) matching [2-4] is to find all sequences such that each of these sequences contains a subsequence $s^{\prime}$, and the distance between $s^{\prime}$ and a query sequence $q$ is at most a given tolerance threshold. In this paper, we focus on accurate matching.

Due to its usefulness in biological sequences, accurate matching have been studied extensively in the literature of bioinformatics. Algorithm $S W$ [15] is known as the first algorithm solving subsequence matching problem in biological sequences. Besides, [16] and [10] proposed more efficient algorithms for accurate matching.

There are a lot of studies about approximate matching [24] requiring users to define a distance metric. [2] used the Euclidean distance as a metric and presented an algorithm for approximate matching by using some indexing techniques. [3] adopted the Dynamic Time Warping (DTW) distance as a metric and proposed an algorithm. [4] proposed to find $k$ sequences with their smallest distances from a given sequence $q$ where the distance metric adopted is the DTW distance.

To the best of our knowledge, no existing studies about sequence matching consider the property table which is studied in this paper.

Since subsequence matching is very useful, it has been commonly adopted in many data mining problems such as frequent subsequence mining $[17,8,18,6$, 5], sequence classification $[13,9,14]$, sequence clustering $[16,15]$, motif detecting $[11,12]$ and subsequence matching $[2,3,15,10]$.

\section{PRoblem Definition}

We are given a set $\mathcal{E}$ of elements. $\mathcal{E}$ is called an element domain set. Each element $e$ is associated with a set $\mathcal{A}$ of $m$ properties or attributes, namely $A_{1}, A_{2}, \ldots, A_{m}$. The value of attribute $A_{i}$ of an element $e$ is denoted by $e . A_{i}$ where $i=1,2, \ldots, m$. In our running example, a set of movies corresponds to the element domain set $\mathcal{E}$. Attribute "Year of Release" and attribute "Director" are two examples of the properties of a movie. For the sake of discussion, we assume that one of the attributes in $\mathcal{A}$ can uniquely identify an element $e$. This attribute is called an identifying attribute. In our example, attribute "Movie Name" is an identifying attribute. Attribute "Director" and attribute "Release Year" are non-identifying attributes.

We define the domain of attribute $A_{i}$, denoted by $D_{i}$, to be the set of all possible values in attribute $A_{i}$ where $i=$ $1,2, \ldots, m$. For instance, all directors like "James Cameron" and "Martin Scorsese" form the domain of attribute "Director". We define the value domain set, denoted by $\mathcal{V}$, to be the union of the domains of all attributes. That is, $\mathcal{V}=\cup_{i=1}^{m} D_{i}$. Note that $\mathcal{V}$ is a set of all possible values. The table (like Table I(b)) containing all attribute values of each element is called the property table.
A value $v$ is said to be an identifying value if $v$ is a value of an identifying attribute. Note that this value $v$ can be used to uniquely identify an element $e$. For the sake of clarity, we simply say that $v$ identifies $e$ (or $e$ is identified by $v$ ). For example, both "Titanic" and "The Aviator" are identifying values but "James Cameron" and "Leonardo DiCaprio" are not. We define $\mathcal{U}$ to be a set of all identifying values. Note that $\mathcal{U} \subseteq \mathcal{V}$. If value $v$ is an identifying value, we define the attribute value set of $v$, denoted by $\alpha(v)$, to be the set of all possible attribute values of the element identified by $v$. For example, if $v$ is "Titanic" (an identifying value), then $\alpha(v)=\{$ "Titanic", 1997, "James Cameron", "Leonardio DiCaprio", "Kate Winslet", ...\}.

A sequence is an ordered list of values where each value is drawn from $\mathcal{V}$. Suppose that there are $k$ values in the sequence, a sequence is represented in form of " $v_{1}, v_{2}, \ldots, v_{k}$ " where $v_{i} \in \mathcal{V}$ for $i=1,2, \ldots, k$. In this representation, for any two values $v_{i}$ and $v_{j}$ where $i<j, v_{i}$ appears before $v_{j}$. "Titanic, The Aviator, Inception" and "Titanic, Leonardo DiCaprio" are two examples of sequences. Consider that $s$ is in form of " $u_{1}, u_{2}, \ldots, u_{l}$ ". Value $u_{i}$ in $s$ is defined to have the temporal position equal to $i$ for $i \in[1, l]$. For example, if $s=$ "Titanic, Leonardo DiCaprio", then "Titanic" has the temporal position equal to 1 and "Leonardo DiCaprio" has the temporal position equal to 2 .

A sequence is said to be an identifying sequence if all values in the sequence are identifying values (i.e., all values in the sequence are drawn from $\mathcal{U}$ ). For example, "Titanic, The Aviator, Inception" is an identifying sequence but "Titanic, Leonardo DiCaprio" is not.

In a lot of applications, we are given a set $S$ of identifying sequences. Let $n$ be the total number of identifying sequences in $S$. In our running example, we are given a set of identifying sequences and each identifying sequence corresponds to the movie rental record of a customer. Table I(a) shows the set of identifying sequences. In the application of finding a researcher, an identifying sequence corresponds to the academic research background of a researcher while in the biology application, it corresponds to a protein sequence.

Let $q$ be a query in form of " $v_{1}, v_{2}, \ldots, v_{k}$ " where $v_{i} \in \mathcal{V}$ for $i \in[1, k]$. Given a value $v \in \mathcal{V}$ and a value $u \in \mathcal{U}, v$ is said to match $u$ if $v \in \alpha(u)$. For example, if $u$ is "Titanic" and $v$ is "Leonardo DiCaprio", then "Leonardo DiCaprio" matches "Titanic" since "Leonardo DiCaprio" $\in \alpha$ ("Titanic").

Definition 1 (Match): Consider a query $q$ in form of " $v_{1}, v_{2}, \ldots, v_{k}$ " where $v_{i} \in \mathcal{V}$ for $i \in[1, k]$. Consider an (identifying) sequence $s \in S$ in form of " $u_{1}, u_{2}, \ldots, u_{l}$ " where $u_{i} \in \mathcal{U}$ for $i \in[1, l]$. Query $q$ is said to match $s$ (or $s$ is matched by $q$ ) if there exist $k$ integers, namely $j_{1}, j_{2}, \ldots, j_{k}$, such that (1) for each $i \in[1, k], v_{i}$ matches $u_{j_{i}}$, and (2) $1 \leq j_{1}<j_{2}<\ldots<j_{k} \leq l$

Consider that $q$ is "Titanic, Leonardo DiCaprio" and $s$ is "Titanic, The Aviator, Inception". Since "The Aviator" was acted by "Leonardo DiCaprio" and "Titanic" occurs before "The Aviator" in $s$, it is easy to verify that $q$ matches $s$. In this example, $k=2$ and $l=3$. We also have $j_{1}=1$ and 

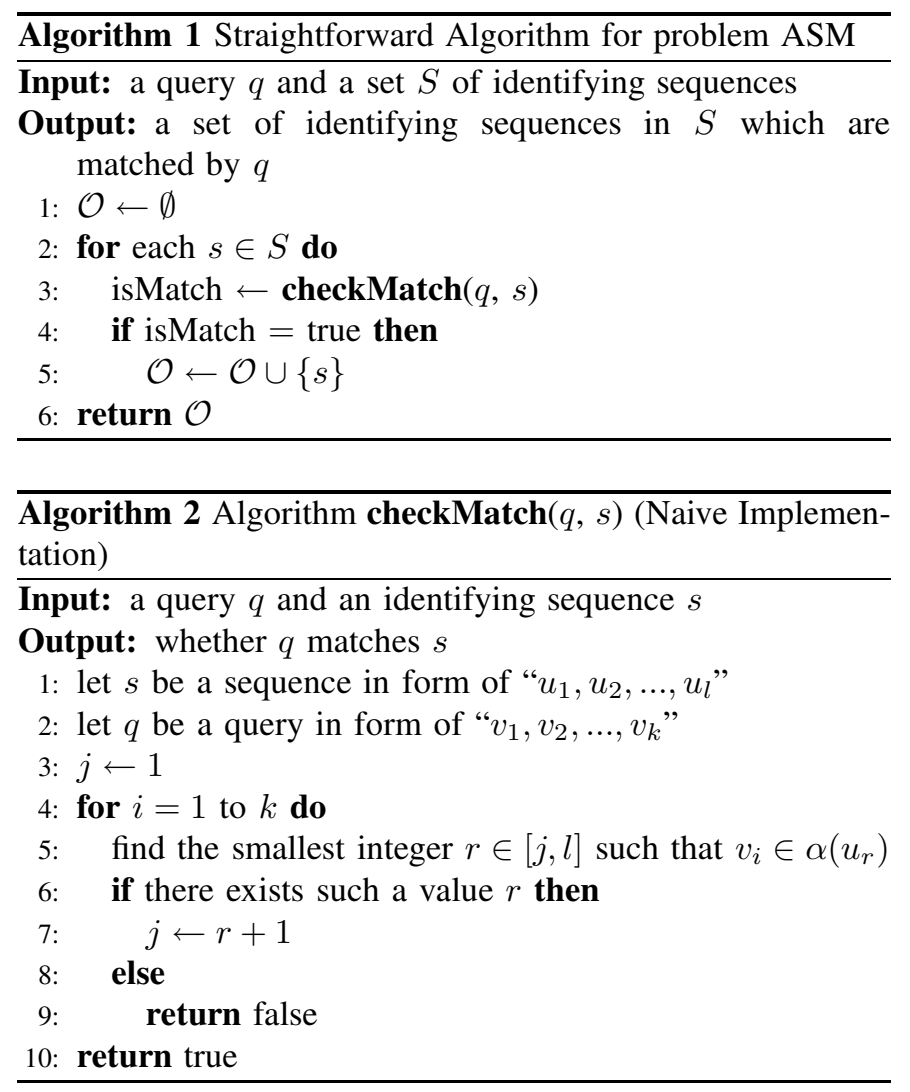

$j_{2}=2$. Note that the first requirement in the above definition holds (i.e., "Titanic" matches itself and "Leonardo DiCaprio" matches "The Aviator"), and the second requirement also holds (i.e., $1 \leq j_{1}<j_{2} \leq l$ ).

In this paper, we are studying the following problem called

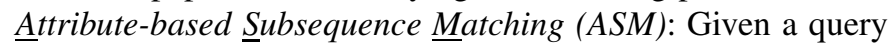
sequence $q$, we want to find all sequences in $S$ which are matched by $q$.

In our running example, if $q$ is "Titanic, Leonardo DiCaprio", we want to find all sequences in $S$ which are matched by $q$. In Table I(a), Alice's sequence is one of the sequences which are matched by $q$.

Note that problem ASM is more general than the traditional problem without considering the property table. This is because problem ASM becomes the traditional problem if there is only one attribute in the property table and this attribute is an identifying attribute.

A possible approach for problem ASM is shown in Algorithm 1. In this algorithm, method $\operatorname{checkMatch}(q, s)$ is to return a boolean value indicating whether a query $q$ matches an identifying sequence $s$. The efficiency of this algorithm depends on how to implement method checkMatch. One naive implementation is shown in Algorithm 2 which takes $O(\mathrm{~lm})$ time where $l$ is the maximum length of a sequence in $S$ and $m$ is the total number of attributes. However, the time complexity of Algorithm 1 is $O(n l m)$ where $n$ is the total number of sequences in $S$. In Section V, we will present an efficient algorithm based on Chinese Remainder Theorem.

\section{Preliminaries}

The remainder for a division of a positive integer $N$ by a positive integer $d$ is denoted by " $N \bmod d$ ". Notation " $\bmod$ " is called a modular operator. Suppose that $N$ is a large number and can be represented by $\mathcal{N}$ 4-byte integers, and $d$ is a small number and can be represented by a 4-byte integer. The time complexity of the modular operation is $O(\mathcal{N})$ [19].

Given a positive integer $N$ and a positive integer $d, d$ is said to be a divisor of $N$ if $(N \bmod d)=0$. Given three positive integers $N, M$ and $d, N$ and $M$ are said to have a common divisor $d$ if $d$ is a divisor of both $N$ and $M$. Two integers $N$ and $M$ are said to be relatively prime if their greatest common divisor is equal to 1 .

The theorems to be introduced are based on the equation using this modular operator in form of $(N \bmod d)=r$ where $N, d$ and $r$ are three positive integers. This equation is called a congruence equation.

We introduce Unique Factorization Theorem. Unique Factorization Theorem is a fundamental theorem in number theory and is widely used in cryptography and security field.

Theorem 1: (Unique Factorization Theorem [19]) Any positive integer $N \geq 1$ can be uniquely expressed as a product of one or more prime numbers called factors of $N$.

Property 1 (Factorization Property [19]): Given a positive integer $N$ and a positive integer $f, f$ is a factor of $N$ if and only if $(N \bmod f)$ is equal to 0 .

Next, we introduce Chinese Remainder Theorem.

Theorem 2: (Chinese Remainder Theorem [19]) Let $m$ be the number of congruence equations. Let $r_{1}, r_{2}, \ldots, r_{m}$ be $m$ positive integers. Suppose that there are $m$ pairwise relatively prime numbers: $n_{1}, n_{2}, \cdots, n_{m}$. Let $N=n_{1} n_{2} \cdots n_{m}$. There exists a unique integer $x \in[0, N-1]$ solving the system of $m$ congruence equations each of which is in form of $(x \bmod$ $\left.n_{i}\right)=r_{i}$ for $i \in[1, m]$.

In the literature, we can compute $x$ by Extended Euclidean algorithm [19] in $O\left(m\left(\log n_{\max }\right)^{2}\right)$ time where $n_{\max }=$ $\max _{i \in[1, m]} n_{i}$. Details can be found in [19].

\section{Modular Algorithm}

We want to design an algorithm called Modular Algorithm with two major requirements which are simple to understand and are used to ease the understanding on how we use Chinese Remainder Theorem for problem ASM. They are Requirement Value Matching and Requirement Sequential Order.

Definition 2: Given a value $v \in \mathcal{V}$ and a sequence $s \in S$, $p(v, s)$ is defined to be a set of all temporal positions such that each of the values at these positions in $s$ is matched by $v$.

Consider Alice's sequence $s$. If $v$ is equal to "Leonardo DiCaprio", then $p(v, s)=\{1,2,3\}$. If $v$ is equal to "Martin Scorsese", then $p(v, s)=\{2\}$.

Consider query $q$ in form of " $v_{1}, v_{2}, \ldots, v_{k}$ ".

Definition 3 (Requirement Value Matching): Let $s$ be a sequence and $q$ be a query in form of " $v_{1}, v_{2}, \ldots, v_{k}$ ". If for each 
TABLE II

LABELS

\begin{tabular}{|c|c||c|c|}
\hline Value & Label & Value & Label \\
\hline Titanic & 2 & The Aviator & 13 \\
\hline 1997 & 3 & 2004 & 17 \\
\hline James Cameron & 5 & Martin Scorsese & 19 \\
\hline Leonardo DiCaprio & 7 & Cate Blanchett & 23 \\
\hline Kate Winslet & 11 & $\ldots$ & $\ldots$ \\
\hline$\ldots$ & $\ldots$ & $\ldots$ & $\ldots$ \\
\hline
\end{tabular}

$i \in[1, k], p\left(v_{i}, s\right) \neq \emptyset$, then $s$ is said to satisfy Requirement Value Matching.

Intuitively, Requirement Value Matching requires that each value in $q$ matches some of the values in a sequence $s$ (without considering the temporal ordering of values). If this requirement is satisfied, we proceed to check the second requirement, Requirement Sequential Order, considering the temporal ordering of values.

Definition 4 (Requirement Sequential Order): Let $s$ be a sequence. If there exist $k$ integers, namely $j_{1}, j_{2}, \ldots, j_{k}$, such that $j_{i} \in p\left(v_{i}, s\right)$ for $i \in[1, k]$ and $j_{1}<j_{2}<\ldots<j_{k}$, then $s$ is said to satisfy Requirement Sequential Order.

Intuitively, Requirement Sequential Order requires that these "matched" values in $s$ have the same temporal ordering as the correspondence values in $q$.

This algorithm involves two major phases. The first phase is called Phase Preprocessing and the second phase is called Phase Query. In Phase Preprocessing, given a set $S$ of identifying sequences and the property table, we generate not only some synopsis of sequences but also some data structures which will be used in Phase Query. In Phase Query, given a query $q$, we find all sequences in $S$ which are matched by $q$ using the information generated in Phase Preprocessing.

Consider a particular sequence $s$ in $S$ and an arbitrary query $q$. We want to create a synopsis for $s$ such that we can determine whether $q$ matches $s$ efficiently using the synopsis. In order to achieve this goal, we should create this synopsis which can help to determine whether the two requirements are satisfied efficiently. In particular, the synopsis contains two separate components. The first component, denoted by $V_{s}$, is used for Requirement Value Matching and corresponds to the first number in this triplet (Section V-A). The other, denoted by $X_{s}$, is used for Requirement Sequential Order and corresponds to the last two numbers in this triplet (Section V-B).

Before we create a synopsis, we first assign each value in $\mathcal{V}$ with a unique prime number called the label of $v$. The label of $v$ is denoted by $P(v)$. Table II shows the labels of some values in $\mathcal{V}$ in our running example.

\section{A. Requirement Value Matching}

Given a sequence $s \in S, V_{s}$ is the value matching number of $s$ which is defined as follows.

Definition 5 (Value Matching Number): Given a sequence $s \in S$ where $s$ is in form of " $v_{1}, v_{2}, \ldots, v_{l}$ ", the value matching number of $s$ is defined to be the product of the labels of all the values in $s$ and their property values. Formally, $V_{s}=\prod_{i=1}^{l} \prod_{v \in \alpha\left(v_{i}\right)} P(v)$.

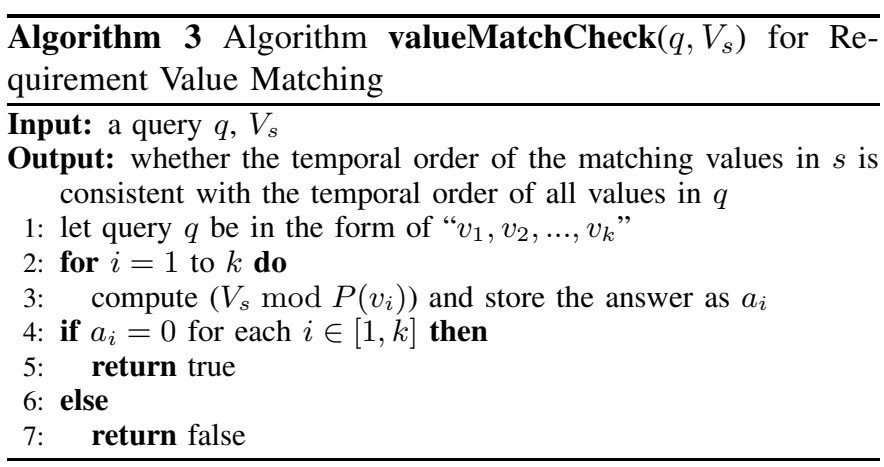

Phase Preprocessing: In Phase Preprocessing, for each sequence $s \in S$, we compute $V_{s}$.

It is easy to verify that the running time of this phase is $O(n l m)$ where $l$ is the greatest length of a sequence.

Phase Query: Recall that Requirement Value Matching is that given a query $q$ and a sequence $s$, each value in $q$ matches one of the values in $s$. By using $V_{s}$, we can perform $k$ modular operations to check whether $s$ satisfies this requirement or not where $k$ is the length of the query sequence. Algorithm 3 shows the algorithm.

With the following lemma, we know that the algorithm is correct.

Lemma 1: Let $s$ be a sequence and $q$ be a query in form of " $v_{1}, v_{2}, \ldots, v_{k}$ ". If $\left(V_{s} \bmod P\left(v_{i}\right)\right)=0$ for each $i \in[1, k]$, then $s$ satisfies Requirement Value Matching.

We know that we can use $V_{s}$ to check for Requirement Value Matching. Now, we want to introduce a stronger requirement called Requirement Duplicate Value Matching. This requirement states that given a sequence $s$ and a query sequence $q$, for each value $v$ in $q$, if $v$ appears $\gamma$ times in $q$ and there exists $\gamma$ values in $s$ such that $v$ matches each of these $\gamma$ values, then $s$ is said to satisfy Requirement Duplicate Value Matching. For example, consider Alice's sequence $s$. If $q=$ "Titanic, Titanic", then $s$ does not satisfy Requirement Duplicate Value Matching. If $q=$ "Leonardo DiCaprio, Leonardo DiCaprio", then $s$ satisfies this requirement.

By using $V_{s}$, we can also check for Requirement Duplicate Value Matching efficiently. By doing this, we modify Algorithm 3 to Algorithm 4.

Now, we analyze the storage size of $V_{s}$. Consider a particular sequence $s$. According to Definition 5, we need to multiply $m l$ prime numbers. In all of our experiments, each prime number can be represented by a 4-byte integer. The storage size of $V_{s}$ is at most $4 \mathrm{ml}$ bytes. In case that a prime number needs more bits for storage, we can use some libraries [20] over large numbers which contain a lot of efficient bitwise operations.

Let the storage size of $V_{s}$ be $\mathcal{N}$. In the above analysis, $4 m l$ is a loose upper bound on $\mathcal{N}$. In other words, in most cases, $\mathcal{N}<<4 m l$. In method valueMatchCheck, it invovles $k$ modular operations where the divisor of each operation is a prime number. Note that in all our experiments, each prime number can be represented by a 4-byte integer. It is easy to 


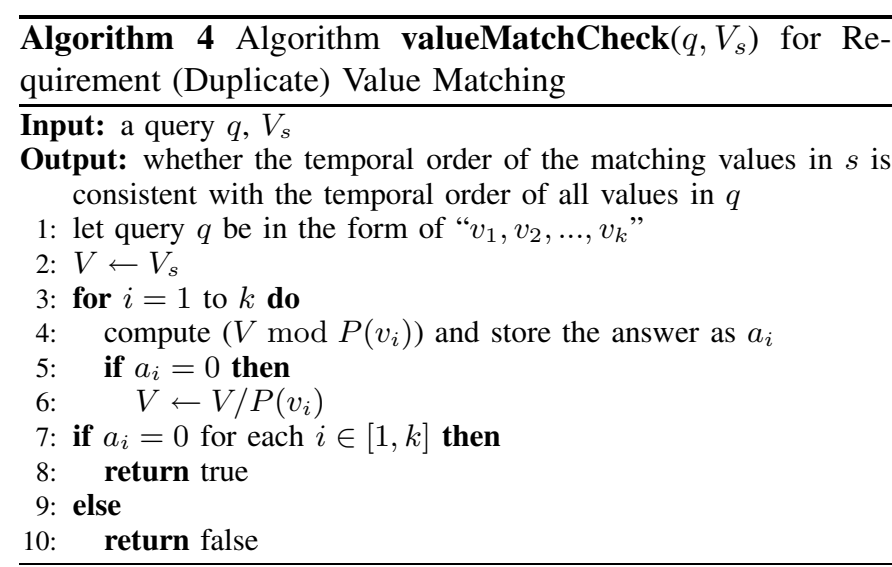

verify that the running time of valueMatchCheck is $O(k \mathcal{N})$.

\section{B. Requirement Sequential Order}

In Section V-B1, we first describe a bulky version of component $X_{s}$. Then, in Section V-B2, we describe a compressed version of component $X_{s}$ by just a pair of two numbers based on Chinese Remainder Theorem.

1) Bulky Version of $X_{s}$ : Phase Preprocessing: Before we describe this component, we give some definitions first.

An interval is defined to be in form of $(l, u)$ where $l$ and $u$ are two positive integers and $l \leq u$.

Definition 6 (Appear After/Before): Let $\triangle_{i}$ and $\triangle_{j}$ be two intervals $\left(l_{i}, u_{i}\right)$ and $\left(l_{j}, u_{j}\right)$, respectively. $\triangle_{i}$ appears before $\triangle_{j}$ (or $\triangle_{j}$ appears after $\triangle_{i}$ ) if $u_{i}<l_{j}$.

Definition 7 (Lifespan): Given a value $v \in \mathcal{V}$ and a sequence $s \in S$ where $p(v, s) \neq \emptyset$, the lifespan of $v$ in $s$, denoted by $L S_{v, s}$, is defined to be an interval in form of $(l, u)$ where (1) $l=\min p(v, s)$ and (2) $u=\max p(v, s)$. We define $L S_{v, s} . l$ to be $l$ and $L S_{v, s} . u$ to be $u$.

Consider Bob's sequence $s$. If $v$ is "Titanic", then $p(v, s)=$ $\{1\}$ and thus the lifespan of $v$ in $s$ is $(1,1)$. If $v$ is "Leonardo DiCaprio", then $p(v, s)=\{1,2\}$ and thus the lifespan of $v$ in $s$ is $(1,2)$.

We are ready to describe the bulky version of $X_{s}$.

Consider a sequence $s$ in $S$. Let $Y$ be the set of all values in $s$ and their property values. Let $h$ be the total number of possible values in $Y$. For each value $v \in Y$, we create an entry in form of $\left(v, L S_{v, s}\right)$. The bulky version of $X_{s}$ is equal to the table storing all these entries.

Example 1: Consider Bob's sequence $s$, "Titanic, The Aviator". Note that "Titanic" has the temporal position equal to 1 and "The Aviator" has the temporal position equal to 2 . The attribute values of "Titanic" are

- "Titanic" (with label = 2),

- "1997" (with label = 3),

- "James Cameron" (with label = 5),

- "Leonardo DiCaprio" (with label = 7) and

- "Kate Winslet" (with label = 11)

The attribute values of "The Aviator" are

- "The Aviator" (with label = 13),
TABLE III

A TABLE SHOWING THE BULKY VERSION OF $X$

\begin{tabular}{|c|c|}
\hline Value $v$ & Lifespan of $v$ in $s$ \\
\hline "Titanic" & $(1,1)$ \\
\hline "1997" & $(1,1)$ \\
\hline "James Cameron" & $(1,1)$ \\
\hline "Leonardo DiCaprio" & $(1,2)$ \\
\hline "Kate Winslet" & $(1,1)$ \\
\hline "The Aviator" & $(2,2)$ \\
\hline "2004" & $(2,2)$ \\
\hline "Martin Scorsese" & $(2,2)$ \\
\hline "Cate Blanchett" & $(2,2)$ \\
\hline
\end{tabular}

- "2004" (with label = 17),

- "Martin Scorsese" (with label = 19),

- "Leonardo DiCaprio" (with label = 7) and

- "Cate Blanchett" (with label = 23)

Thus, we have the set $Y$ equal to \{"Titanic", "1997", "James Cameron", "Leonardo DiCaprio", "Kate Winslet", "The Aviator", "2004", "Martin Scorsese", "Cate Blanchett" \}. Then, we calculate the lifespan of each value $v \in Y$ in this sequence as shown in Table III. For example, if $v=$ "Titanic", then the lifespan of $v$ in $s$ is $(1,1)$. Similarly, if $v=$ "Leonardo DiCaprio", then the lifespan of $v$ in $s$ is $(1,2)$. Table III corresponds to the bulky version of $X_{s}$.

Let $l$ be the greatest length of a sequence in $S$. There are $O(l m)$ possible values in $Y$. Thus, the size of the bulky version of $X_{s}$ is $O(\mathrm{~lm})$. In Section V-B2, we will present a compressed version of $X_{s}$ which contains only two positive numbers. Similarly, we can easily derive that the complexity of this phase is $O(\mathrm{~lm})$.

Phase Query: Suppose that we are given a query sequence $q$ and a sequence $s$ in $S$. We want to check whether $q$ matches $s$ using the bulky version of $X_{s}$.

Definition 8 (Query-Aware Lifespan): Let $s$ be a sequence in $S$ and $X_{s}$ be the bulky version of $X_{s}$ for $s$. Given a query sequence $q$ in form of $\left(v_{1}, v_{2}, \ldots, v_{k}\right)$, the query-aware lifespan of $s$ with respect to $q$, denoted by $Q A-L S(s, q)$, is defined to be $\left(\triangle_{1}, \triangle_{2}, \ldots, \triangle_{k}\right)$ where $\triangle_{i}$ is the lifespan of $v_{i}$ in $s$ for $i \in[1, k]$.

Our strategy is to create the query-aware lifespan of $s$ with respect to $q$ according to the bulky version of $X_{s}$. This can be done in $O(k)$ time if the bulky version of $X_{s}$ is indexed with a hash data structure. Then, according to the query-aware lifespan, we can determine whether $q$ matches $s$ efficiently, which will be described next.

Definition 9 (Non-Overlapping): Consider a sequence $s$ and a query sequence $q$. Let the query-aware lifespan of $s$ with respect to $q$ be $\left(\triangle_{1}, \triangle_{2}, \ldots, \triangle_{k}\right)$. The query-aware lifespan is said to be non-overlapping if and only if for each $i, j \in[1, k]$ where $i<j, \triangle_{i}$ appears before $\triangle_{j}$.

Definition 10 (Invalid): Consider a sequence $s$ and a query sequence $q$. Let the query-aware lifespan of $s$ with respect to $q$ be $\left(\triangle_{1}, \triangle_{2}, \ldots, \triangle_{k}\right)$. The query-aware lifespan is said to be invalid if and only if there exist any two integers $i, j \in[1, k]$ such that $i<j$ and $\triangle_{i}$ appears after $\triangle_{j}$.

With the above definitions, we have the following lemma about how to determine whether $q$ matches $s$ or not. 


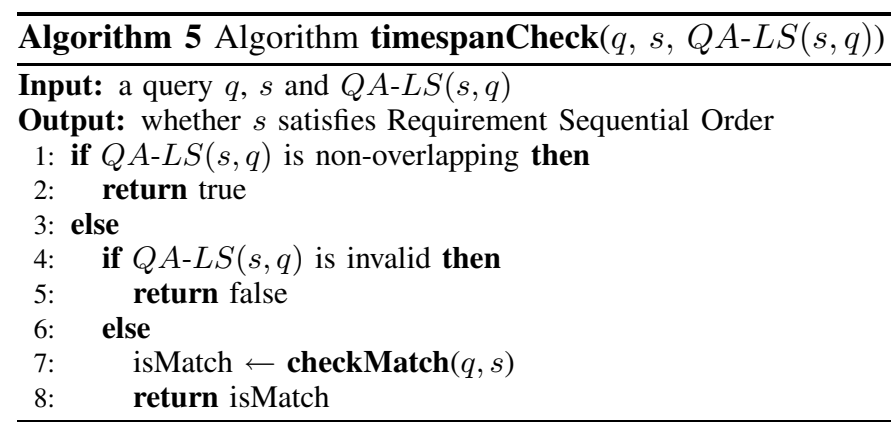

Lemma 2: Consider a sequence $s$ and a query sequence $q$. If the query-aware lifespan of $s$ with respect to $q$ is nonoverlapping, then $q$ matches $s$. If the query-aware lifespan of $s$ with respect to $q$ is invalid, then $q$ does not match $s$.

Suppose that we are given the query-aware lifespan of $s$ with respect to $q$. Algorithm 5 shows the steps of checking whether $q$ matches $s$ according to the query-aware lifespan only. It is easy to verify that checking the conditions on whether the query-aware lifespan is non-overlapping (or invalid) takes $O(k)$ time. If these conditions are not satisfied, we need to execute the statements in lines 7-8 involving checkMatch which takes $O(\mathrm{~lm})$ time. In our experiments, on average, there are about $90 \%$ cases that the query-aware lifespan is either non-overlapping or invalid. Thus, in most cases, the running time of sequentialOrderCheck is $O(k)$.

Let us analyze the storage complexity of the bulky version of $X_{s}$. Consider a particular sequence $s$. Let $|Y|$ be the average size of $Y$ (i.e., the average number of possible values in a sequence $s$ and their property values). For each value $v \in Y$, we need to store entry $\left(v, L S_{v, s}\right)$. In our implementation, $v$ is stored in form of a prime number and $L S_{v, s}$ is stored in form of two temporal positions. Since in all our experiments, the greastest possible values of each prime number and each temporal position can be represented by a 4-byte integer, each prime number and each temporal position are stored in a 4byte integer. Thus, each entry occupies $4 \times 3=12$ bytes. Since there are $|Y|$ entries, the storage size of the bulky version of $X_{s}$ for a particular sequence $s$ is equal to $12|Y|$ bytes.

2) Compressed Version of $X_{s}$ Based on a Pair of Numbers: Consider a sequence $s$ in $S$. The bulky version contains $h$ entries and each entry contains a value and its lifespan in $s$. This bulky version occupies a lot of space. Interestingly, the compressed version of $X_{s}$ to be described contains only two positive numbers, which is quite space-efficient.

Specifically, given a sequence $s \in S$, the compressed version of $X_{s}$ is defined to be equal to a pair of two numbers. The first number is called the lower-bound sequential order number of $s$, denoted by $L_{s}$, and the second number is called the upper-bound sequential order number of $s$, denoted by $U_{s}$. In Phase Preprocessing, these two numbers are to be found.

Phase Preprocessing: In Phase Preprocessing, for each sequence $s \in S$, we compute $L_{s}$ and $U_{s}$ as follows.

Let $Y$ be the set of all values in $s$ and their property values. Let $h$ be the total number of possible values in $Y$. Recall that in the bulky version, for each value $v \in Y$, we create an entry in form of $\left(v, L S_{v, s}\right)$ where $L S_{v, s}$ is the lifespan of $v$ in $s$. Note that $L S_{v, s}$ is in form of $\left(L S_{v, s} . l, L S_{v, s} . u\right)$. However, in the compressed version, for each value $v \in Y$, we conceptually create a pair of congruence equations as follows. The following equations are in the format of Chinese Remainder Theorem. Note that $P(v)$ is the label of value $v$.

$$
\begin{aligned}
& \left(L_{s} \bmod P(v)\right)=L S_{v, s} \cdot l \\
& \left(U_{s} \bmod P(v)\right)=L S_{v, s} . u
\end{aligned}
$$

Since we have $h$ values in $Y$, we conceptually generate $h$ congruence equations in form of (1) and $h$ congruence equations in form of (2).

We first consider the $h$ equations for $L_{s}$ in form of (1) and describe how to determine $L_{s}$, one of the two numbers stored in the compressed form of $X_{s}$. Specifically, since the labels of all values in $Y$ are prime numbers, they are pairwise relatively prime. Note that $P(v)$ and $L S_{v, s} . l$ are given in Equation (1) where $v \in Y$. This is the equation format of Chinese Remainder Theorem. By using the Extended Euclidean algorithm, we can find a unique integer $L_{s} \in[0, N-1]$ where $N$ is the product of the labels of all values in $Y$.

We can use a similar technique to find $U_{s}$ by considering the $h$ equations for $U_{s}$ in form of (2).

Thus, we obtain that the final compressed version of $X_{s}$ are two numbers, namely $L_{s}$ and $U_{s}$.

Example 2: Consider Bob's sequence again. According to Example 1, we can obtain the bulky version of $X_{s}$ (Table III).

In the compressed version, since we have 9 values in $Y$, we can conceptually formulate 9 congruence equations for $L_{s}$ and 9 congruence equations for $U_{s}$. Take $v=$ "Titanic" for illustration. Since its label is equal to 2 and its $L S_{v, s} . l$ is equal to 1, according to Equation (1), we create

$$
\left(L_{s} \bmod 2\right)=1
$$

The other 8 congruence equations for $L_{s}$ are:

$$
\begin{array}{lll}
\left(L_{s} \bmod 3\right)=1 & \left(L_{s} \bmod 11\right)=1 & \left(L_{s} \bmod 19\right)=2 \\
\left(L_{s} \bmod 5\right)=1 & \left(L_{s} \bmod 13\right)=2 & \left(L_{s} \bmod 23\right)=2 \\
\left(L_{s} \bmod 7\right)=1 & \left(L_{s} \bmod 17\right)=2 &
\end{array}
$$

Similarly, we can construct the 9 congruence equations for $U_{s}$. By the Extended Euclidean Algorithm, we obtain $L_{s}=$ $134,918,071$ and $U_{s}=7,436,431$.

In Section IV, we know that the time complexity of finding a solution for $L_{s}$ (and $U_{s}$ ) with $m$ congruence equations is $O\left(m\left(\log n_{p}\right)^{2}\right)$ time where $n_{p}$ is the largest prime numbers we use. Since each sequence $s$ is associated with $L_{s}$ (and $U_{s}$ ), the running time of this phase considering all sequences is equal to $O\left(n m\left(\log n_{p}\right)^{2}\right)$.

Phase Query: Suppose that sequence $s$ satisfies Requirement Value Matching. Recall that Requirement Sequential Order is that the "matched" values in $s$ have the same temporal ordering as the correspondence values in $q$. Algorithm 6 shows how we check whether $s$ satisfies Requirement Sequential Order using 


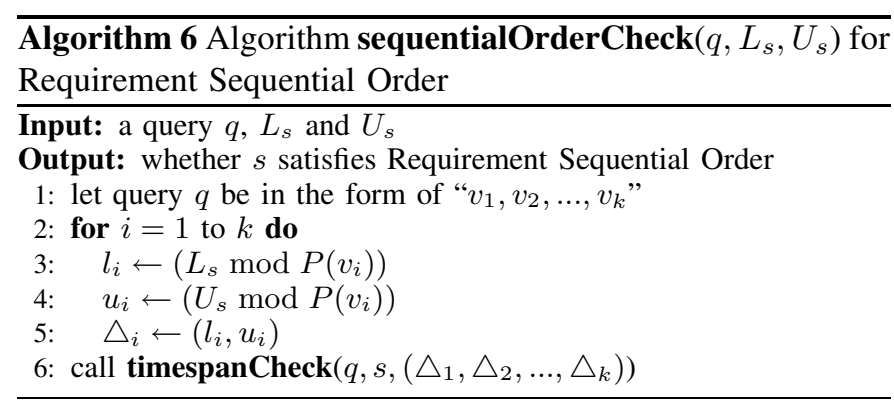

$L_{s}$ and $U_{s}$. It is easy to see Algorithm 6 returns a correct solution with the following lemma.

Lemma 3: Consider a sequence $s$. Let $L_{s}$ and $U_{s}$ be the lower-bound sequential order number and the upper-bound sequential order, resepectively. Let $Y$ be the set of all values in $s$ and their property values. Given a value $v \in Y$, the lifespan of $v$ in $s$ is equal to $(l, u)$ where $l=\left(L_{s} \bmod P(v)\right)$ and $u=\left(U_{s} \bmod P(v)\right)$.

Let us analyze the storage complexity of the compressed version of $X_{s}$. Consider a particular sequence $s$. For this sequence $s$, we need to store two numbers, namely $L_{s}$ and $U_{s}$. Consider number $L_{s}$ which is computed based on $|Y|$ congruence equations. Note that $L_{s}$ is at most the multiplication of the divisors of all congruence equations (i.e., $\prod_{v \in Y} P(v)$ ). In all of our experiments, each divisor (or each prime number) can be represented by a 4-byte integer. Thus, $L_{s}$ can be represented by $|Y|$ 4-byte integers and thus the size of $L_{s}$ is at most $4|Y|$ bytes. Note that $4|Y|$ is an upper bound of the size of $L_{s}$. In most cases, the exact size is smaller than $4|Y|$. Similarly, we can derive that the size of $U_{s}$ is at most $4|Y|$ bytes. The storage size of the compressed version of $X_{s}$ given a particular sequence is at most $4|Y|+4|Y|=8|Y|$ bytes. Since $|Y| \leq m l$, the storage size is at most $8 \mathrm{ml}$ bytes.

Let $\mathcal{N}^{\prime}$ be the storage size of $L_{s}$ (or $U_{s}$ ). Similarly, in the above analysis, $4|Y|$ is a loose upper bound on $\mathcal{N}^{\prime}$. In other words, $\mathcal{N}^{\prime}<<4|Y|(<4 m l)$. It is easy to verify that the running time of sequentialOrderCheck is $O\left(k \mathcal{N}^{\prime}\right)$ time if the running time of timespanCheck is $O(k)$ in most cases.

3) Comparison: Let us compare the storage of the compressed version with the storage of the bulky version. Consider $X_{s}$ of both versions. The compressed version of $X_{s}$ occupies at most $8|Y|$ bytes and the bulky version of $X_{s}$ occupies $12|Y|$ bytes. Thus, the storage size of the compressed version of $X_{s}$ is at most $2 / 3$ of the bulky version of $X_{s}$. Now, we consider the storage size of the compressed/bulky synopsis containing not only $X_{s}$ but also $V_{s}$. Note that $V_{s}$ is the common component used by the compressed version and the bulky version. Note that since the storage size of $X_{s}$ is equal to $4 \mathrm{ml}$, the compressed synopsis occupies at most $4 m l+8|Y| \leq 12 m l$ bytes and the bulky synopsis occupies $4 m l+12|Y| \leq 16 m l$. Thus, the storage size of the compressed synopsis is at most $3 / 4(=12 / 16)$ of the storage size of the bulky synopsis. In the experimental results (Section VII), the real compression effect is more significant. On average, the storage size of the compressed synopsis is about $1 / 4$ of

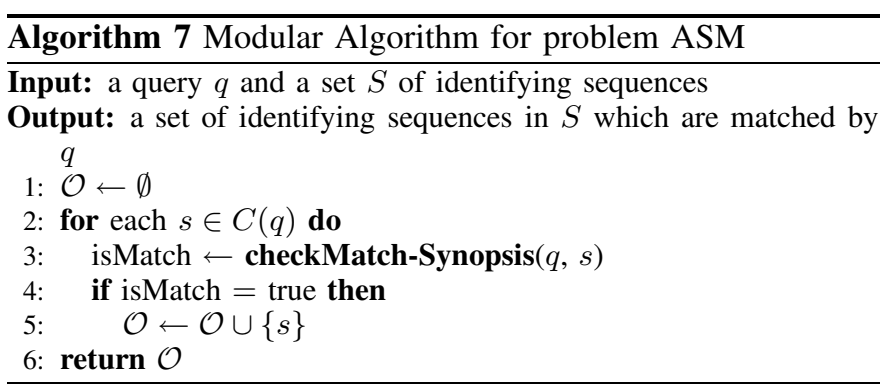

the storage size of the bulky synopsis. The above theoretical analysis is based on the upper bound of the storage size of the compressed synopsis (instead of the exact storage size) and thus the bound of $3 / 4$ is not quite tight.

\section{Putting Two Requirements Together}

In this section, we present algorithms to combine the two requirements together in addition to introducing an indexing technique called inverted list.

1) Phase Preprocessing: In addition to the steps we discussed previously, we describe an indexing technique called inverted list.

Suppose that each sequence $s \in S$ is given a unique sequence ID. Given a value $v \in \mathcal{V}$, the inverted list of $v$, denoted by $I(v)$, is defined to be a set of sequence IDs such that one of the values in each of these sequences has its property values equal to $v$. Given a query $q$, we define $C(q)$ to be a set of sequence IDs where each of the sequences with these IDs satisfies Requirement Value Matching. Thus, $C(q)$ is equal to $\cap_{i=1}^{k} I\left(v_{i}\right)$.

So, there are two major steps in Phase Preprocessing. The first step is to generate the inverted list of $v$ for each value $v \in \mathcal{V}$. The second step is to generate the synopsis of each sequence $s$ (where the synopsis is in form of a triplet $\left.\left(V_{s}, L_{s}, U_{s}\right)\right)$.

Note that both component $V_{s}$ described in Section V-A and inverted lists are used for Requirement Value Matching. However, there are some differences. Firstly, inverted lists are used to locate sequences satisfying Requirement Value Matching by sequence IDs. Secondly, $V_{s}$ can be used for Requirement Duplicate Value Matching but inverted lists cannot.

Note that the complexity of synopsis generation is $O(\mathrm{nlm})$ where $l$ is the greatest length of a sequence. Generating inverted lists also takes $O(\mathrm{nlm})$ time. The overall complexity of this phase is equal to $O(n l m)$.

2) Phase Query: With the inverted list, we can modify Algorithm 1 to Algorithm 7. The differences come from the statements in Line 2 and Line 3. Firstly, in Line 2 of Algorithm 7, instead of processing all sequences in $S$, we process the sequences in $C(q)$ using the inverted list. Secondly, in Line 3 of Algorithm 7, instead of calling the original method checkMatch without using any synopsis, we call the new method checkMatch-Synopsis using the synopsis.

Algorithm 8 shows the algorithm for checkMatchSynopsis. With Lemmas 1, 2 and 3, it is easy to verify the following theorem. 


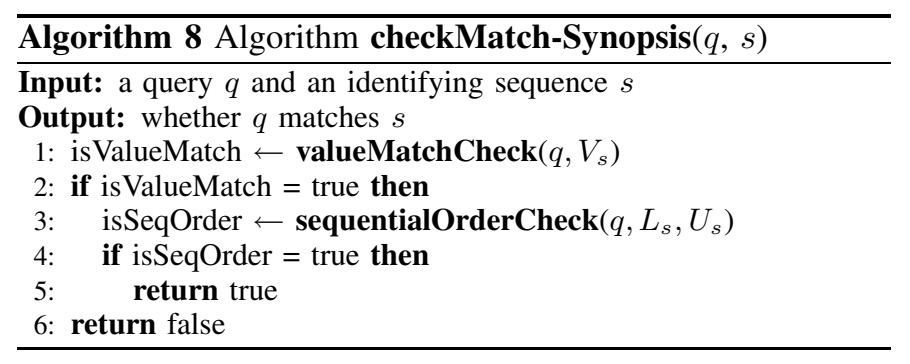

Theorem 3: Algorithm 7 returns all sequences which are matched by $q$.

Consider Algorithm 7. There are $O(n)$ sequences in $C(q)$. Consider a sequence in $C(q)$. We need to execute checkMatch-Synopsis (Algorithm 8). In this algorithm, we know that valueMatchCheck takes $O(k \mathcal{N})$ time and sequentialOrderCheck $\left(q, L_{s}, U_{s}\right)$ takes $O\left(k \mathcal{N}^{\prime}\right)$ time in most cases. Thus, checkMatch-Synopsis takes $O\left(k\left(\mathcal{N}+\mathcal{N}^{\prime}\right)\right)$ time. Since $\mathcal{N} \geq \mathcal{N}^{\prime}$, the time complexity of checkMatch-Synopsis becomes $O(k \mathcal{N})$. In conclusion, the overall time complexity of Algorithm 7 is $O(n k \mathcal{N})$.

\section{Frequent Subsequence Mining}

In this section, we introduce a data mining problem, frequent attribute-based subsequence mining, which frequently makes use of the efficient operator for ASM (i.e., checking whether a query sequence matches a sequence in the dataset). Traditional frequent subsequence mining has been studied extensively in the literature $[17,8,18,6,5]$. It is useful to find frequent patterns in order to study customers' behaviors and temporal patterns. In this section, we propose frequent attribute-based subsequence mining (FASM). It is the same as the traditional mining except that we consider the property table.

Given a sequence $p$ in form of " $v_{1}, v_{2}, \ldots, v_{x}$ " where $v_{i} \in \mathcal{V}$ for $i \in[1, x]$, the frequency of $p$ is defined to be the total number of sequences in $S$ which are matched by $p$. Given a parameter $\theta$ which is a positive integer and a user parameter, a sequence $p$ is said to be frequent if the frequency of $p$ is at least $\theta$. The problem of finding frequent attribute-based subsequence mining (FASM) is: given a parameter $\theta$, we want to find all possible frequent sequences in $S$.

There are at least two categories of finding frequent subsequence mining in the literature. The first category is singletonbased mining while the second category is set-based mining.

In singleton-based mining, a sequence is represented in form of " $u_{1}, u_{2}, \ldots, u_{l}$ " where $u_{i} \in \mathcal{V}$ for $i \in[1, l]$. At each timestamp, there is only at most one value $\in \mathcal{V}$ in the subsequence [1]. Singleton-based mining is to find all frequent subsequences in the dataset which have their frequencies at least a given threshold $\theta$.

In set-based mining, a sequence is represented as a setsequence in form of " $G_{1}, G_{2}, \ldots, G_{l}$ " where $G_{i} \subseteq \mathcal{V}$ for $i \in$ $[1, l][21,8]$. At each timestamp, there can be more than one value $\in \mathcal{V}$ in the set-sequence (which is represented by a set $G_{i}$ instead of a value in $\mathcal{V}$ ) [1]. In this category, the concept of subsequence (or set-subsequence) is defined differently as follows. Given a set-sequence $g$ in form of " $G_{1}, G_{2}, \ldots, G_{l}$ " where $G_{i} \subseteq \mathcal{V}$ for $i \in[1, l]$ and another set-sequence $h$ in form of " $H_{1}, H_{2}, \ldots, H_{l^{\prime}}$ " where $H_{i} \subseteq \mathcal{V}$ for $i \in\left[1, l^{\prime}\right], g$ is said to be a set-subsequence of $h$ if there exist $l$ integers, namely $j_{1}, j_{2}, \ldots, j_{l}$, such that (1) for each $i \in[1, l], G_{i} \subseteq H_{j_{i}}$, and (2) $1 \leq j_{1}<j_{2}<\ldots<j_{l} \leq l^{\prime}$. If $g$ is a set-subsequence of $h$, then $h$ is said to contain $g$. In set-based mining, each set-sequence in the dataset is in form of " $G_{1}, G_{2}, \ldots, G_{l}$ ". The frequency of a set-sequence $g$ is equal to the total number of set-sequences in the dataset containing $g$. Set-based mining is to find all frequent set-subsequences in the dataset which have their frequencies at least a given threshold $\theta$.

Note that our FASM problem is a special case of the setbased mining. That is, all sequences found in our FASM problem can be found in the set of set-sequences found in the set-based mining.

Since our FASM problem is a special case of the set-based mining, we adapt an existing algorithm in the literature of setbased mining for FASM, by using our efficient query operator in ASM. In the literature, most algorithms for set-based mining requires to enumerate some potential candidates as the output and count the total number of sequences in the dataset which are matched by each candidate (query sequence). Our operator for ASM can be used in the counting step. Whenever we need to obtain the count for each candidate, we can perform our operator. Since these algorithms involves a large set of candidates and their counting step is not optimized, if their counting step is replaced by our operator, the efficiency of the algorithms can be improved a lot. In the experiment, we use the algorithm in [8] to illustrate how the operator can improve the performance of the algorithm.

Our operator can also be used to solve a more general problem, the set-based mining, using the above approach. For each candidate in form of " $G_{1}, G_{2}, \ldots, G_{l}$ " where $G_{i}$ is a set of values for $i \in[1, l]$, we generate all possible sequences in form of " $v_{1}, v_{2}, \ldots, v_{l}$ " where $v_{i} \in G_{i}$ for $i \in[1, l]$. Each generated sequence can be regarded as a query in ASM.

\section{EMPIRICAL EXPERIMENTS}

In order to verify the efficiency of our algorithm, we implemented three algorithms in $\mathrm{C} / \mathrm{C}++$, namely Naive, $M A$ and MAI. In Section III, we described two possible straightforward approaches for problem ASM. Since the first approach takes an exponential time with respect to the length of the query length which is not scalable, we implemented the second straightforward approach (Algorithm 1) and call it Naive. $M A$ is Modular Algorithm without inverted list which is Algorithm 7 where $C(q)$ in line 2 is replaced by $S$. MAI is Modular Algorithm with inverted list which is Algorithm 7. For $M A$ and $M A I$, we adopt the compressed version of the synopsis because it occupies less storage and have nearly the same execution time in Phase Preprocessing and Phase Query compared with the bulky version.

All the experiments were performed on a $2.4 \mathrm{GHz} \mathrm{PC}$ with 4.0GB RAM, on a Linux platform. We did experiments on both synthetic and real datasets. For the synthetic datasets, 
TABLE IV

DEFAULT VALUES

\begin{tabular}{|c|c|}
\hline Parameters & Values \\
\hline$n$ (Number of sequences) & $\mathbf{2 5 0 k}, 500 \mathrm{k}, 750 \mathrm{k}, 1 \mathrm{M}$ \\
\hline$\mu$ (Average sequence length) & $20, \mathbf{4 0}, 60,80,100$ \\
\hline$k$ (Query length) & $5, \mathbf{1 0}, 15,20$ \\
\hline$d$ (Domain size of each attribute) & $\mathbf{1 0 0}, 200,30,40$ \\
\hline$m$ (Number of attributes) & $\mathbf{5}, 10,15,20$ \\
\hline
\end{tabular}

we first generate the length of the sequence following a given Gaussian distribution with its mean equal to $\mu$ and its standard derivation equal to 5 where $\mu$ is a user parameter representing the average length of a sequence. In addition to dataset size $n$ and the average length of each sequence $\mu$, the synthetic data generator also simulates the number of attributes $m$, the size of each attribute domain $d$ and the length of each query $k$. We assume these three values are fixed for all the sequences in a single dataset. In order to find at least one matching in the whole dataset, we extract each query sequence from an arbitrary sequence in the dataset. The values of each parameter used in the experiments are given in Table IV, where the default values are in bold. Finally, we generate the synthetic datasets according to every distinct parameter setting in Table IV.

In the experiments, we evaluate the algorithms with four measurements: (1) Preprocessing Time, (2) Execution Time, (3) Storage and (4) Compression Ratio. (1) Preprocessing time of $M A$ and MAI corresponds to the time cost in Phase Preprocessing. Naive has no preprocessing step. So, we do not consider it. (2) Query time refers to the time an algorithm takes to answer 100 queries. Since the query time of $M A(M A I)$ using the compressed synopsis is similar to the query time of $M A$ (MAI) using the bulky synopsis, we only report MA (MAI) using the compressed synopsis. (3) Storage is the total memory consumption used for each data structure and the original dataset. The storage of Dataset Size is the memory occupied by the sequence data. The storage of Inverted List is the memory occupied by the inverted list. The storage of Compressed Synopsis is the memory occupied by the compressed version of $X_{s}$ and $V_{s}$, while the storage of Bulky Synopsis is the storage occupied by the bulky version of $X_{s}$ and $V_{s}$. (4) Compression Ratio is the ratio of the storage of Compressed Synopsis to that of Bulky Synopsis.

We study the effects of $n, \mu, k, d$ and $m$ as follows.

Effect of database size $n$ : Figure 1(a) shows that the preprocessing in $M A I$ is slightly larger than that of $M A$. It is because that $M A I$ needs to generate the inverted list, but $M A$ does not. In Figure 1(b), when the execution time of Naive increased sharply when $n$ increases, the execution time of $M A$ and MAI increased slightly. As expected, the storage of Bulky Synopsis is much larger than that of Dataset Size, Inverted List and Compressed Synopsis in Figure 1(c). The compression ratio is around $24 \%$ as shown in Figure 1(d).

Effect of sequence length $\mu$ : Figures 2(a), (b) and (c) have similar trends as Figures 1(a), (b) and (c). In Figure 2(c), each data structure increases with $\mu$. Note that the compressed synopsis also increases. In the compressed synopsis, each sequence is compressed into a 3 -number synopsis which is independent of the sequence length. So, apparently, it seems that it should not increase with the sequence length. However, when the sequence becomes longer, the compressed synopsis needs much larger prime numbers and thus the synopsis representation needs more storage. Notice that, in Figure 2(d), as $\mu$ increased, the compression ratio decreased slowly, which means the longer the sequence is, the smaller storage the compressed synopsis occupies compared with the bulky synopsis.

Effect of $k$ : As expected, the length of the query sequence does not affect the processing time and the storage of every data structure, as shown in Figure 3(a), Figure 3(c) and Figure 3(d). From Figure 3(b), we can see that the execution time of $M A$ and $M A I$ remained unchanged while that of Naive increased slightly.

Effect of $d$ : As $d$ increases, the storage of the compressed synopsis increased slightly, so that the compression ratio also increased, as shown in Figure 4(c) and Figure 4(d). When $d$ increased, the diversity of the sequences in the database also increased sharply, each generated sequence is much more dissimilar to other sequences. Consequently, the execution time of $M A$ and $M A I$ decreased in Figure 4(b).

Effect of $m$ : When $m$ increased, the processing time and storage increased, as shown in Figure 5(a) and Figure 5(c). But the compression ratio remained around $24 \%$ in Figure 5(d). But in Figure 5(b), the execution time of MAI remained when $m$ increases. However, the execution time of Naive increased significantly. It means that $M A I$ can deal with sequences that have a large number of attributes efficiently.

Besides the synthetic datasets, we also did experiments on three real datasets: Netflix [22], BookX [23], and Genealogy [24]. (1) Netflix is a famous movie rental company. We process the rating record dataset provided by Netflix to generate a rating sequence dataset through grouping the ratings by customerID (the identification of a customer) and sorting them by the rating date. (2) BookX (BookCrossing) is an online book searching and rating website. We download the ratings dataset and use a similar method to generate a sequence for every reader. (3) Genealogy dataset is collected by ourselves, which contains biographic sequences of 1000 researchers. Some statistics of the three datasets are shown in Table V. Each query is generated by randomly selecting a subsequence of a sequence in a real dataset such that the length of the subsequence is equal to a specified length. generated by randomly selecting subsequences of sequences in each In this table, No. of Elements is the number of elements appearing in this dataset, and Avg. Duplicates is the average proportion of duplicate attribute values in one sequence.

The first four columns in Table VI shows the execution time on the three real datasets. The execution time of MAI is much smaller than that of Naive in every real dataset. The last column in Table VI shows that the compression ratio of Netflix and BookX is around 30\%, a little higher than that of the synthetic datasets. We summarize the other statistics of the experiments on real datasets. The greatest prime numbers used in encoding the three real datasets is $4,863,427$. The greatest number used in the compressed synopsis contains 2100 digits. 


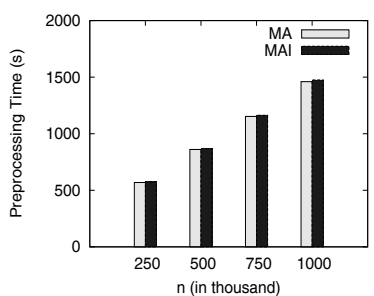

(a)

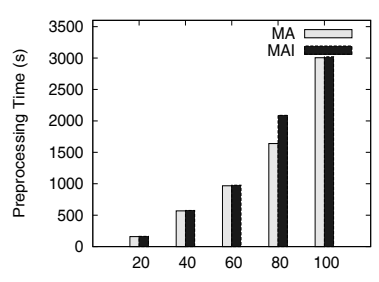

(a)

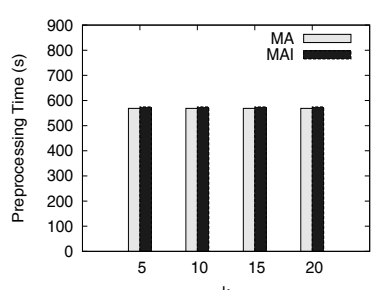

(a)

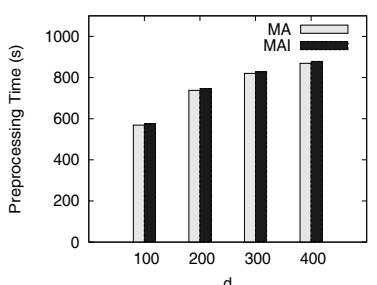

(a)

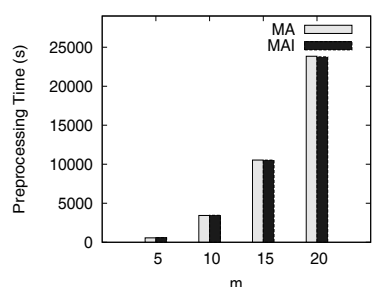

(a)

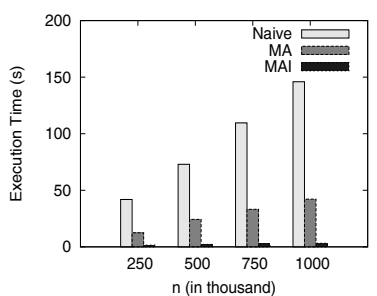

(b)

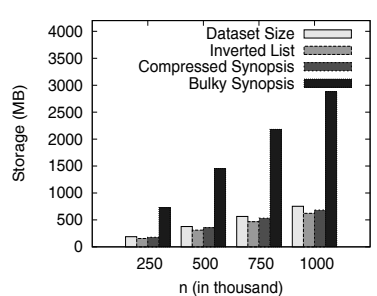

(c)

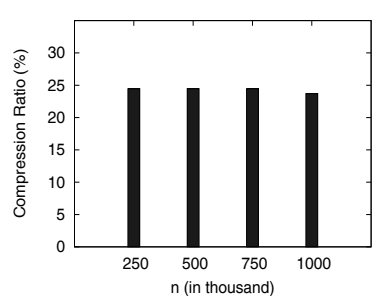

(d)

Fig. 1. Effect of $n$ (Dataset size)

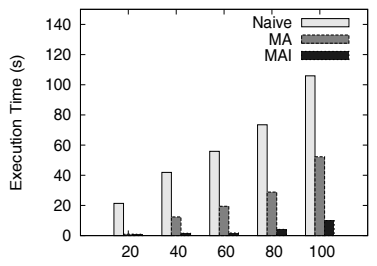

(b)

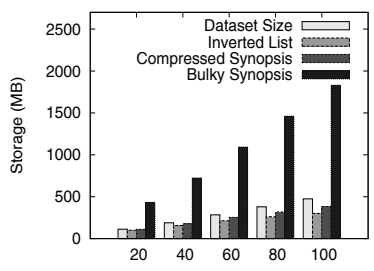

(c)

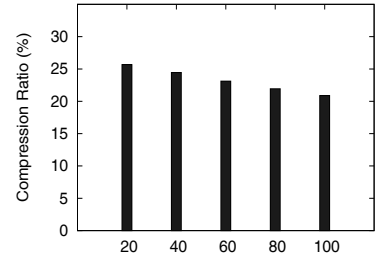

(d)

Fig. 2. Effect of $\mu$ (Average length of a sequence)

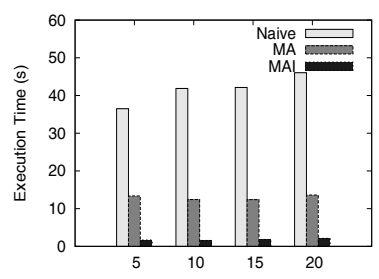

(b)

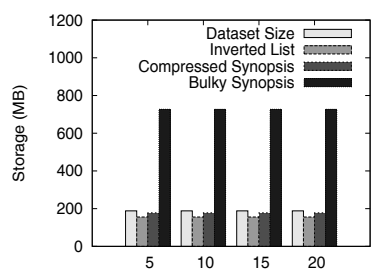

(c)

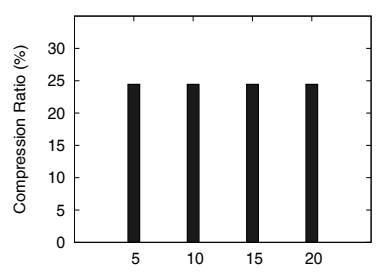

(d)

Fig. 3. Effect of $k$ (Length of a query sequence)

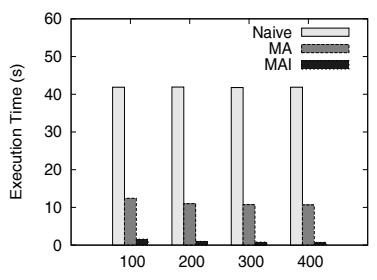

(b)

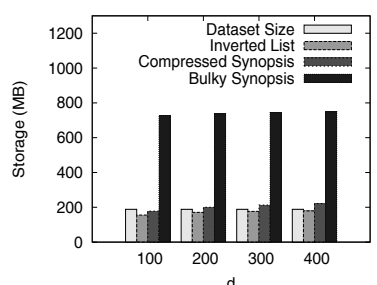

(c)

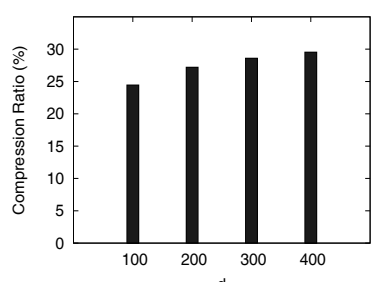

(d)

Fig. 4. Effect of $d$ (Size of the domain of each attribute)

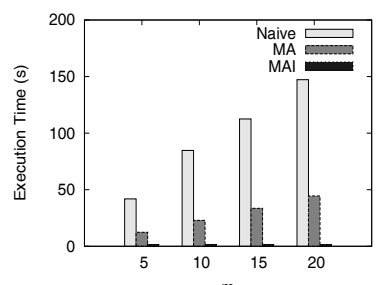

(b)

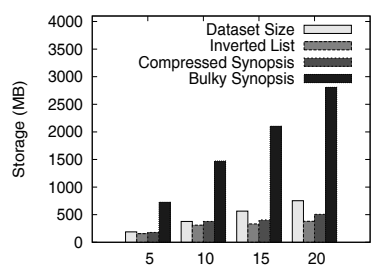

(c)

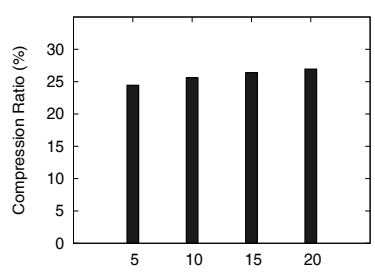

(d)

Fig. 5. Effect of $m$ (Number of attributes of each object)

TABLE V

StATISTICS OF REAL DATASETS

\begin{tabular}{|c|c|c|c|c|c|}
\hline Datasets & $n$ & $m$ & $\mu$ & No. of Elements & Avg. Duplicates \\
\hline Netflix & 478905 & 3 & 157 & 7653 & $11.2 \%$ \\
\hline BookX & 91400 & 3 & 11.7 & 262554 & $4.2 \%$ \\
\hline Genealogy & 1000 & 3 & 2.5 & 1553 & $13.3 \%$ \\
\hline
\end{tabular}

TABLE VI

EXECUTION TIME ON REAL DATASETS

\begin{tabular}{|c|c|c|c|c|}
\hline \multirow{2}{*}{ Dataset } & \multicolumn{3}{|c|}{ Execution Time (s) } & \multirow{2}{*}{$\begin{array}{c}\text { Compression } \\
\text { Ratio (\%) }\end{array}$} \\
\hline & Naive & MA & MAI & \\
\hline Netflix & 205.939 & 85.446 & 6.744 & 31.382 \\
\hline BookX & 2.489 & 2.034 & 0.681 & 30.978 \\
\hline Genealogy & 0.011 & 0.004 & 0.003 & 21.699 \\
\hline
\end{tabular}




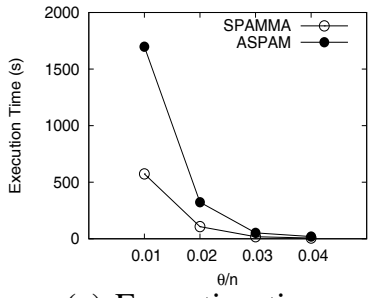

(a) Execution time

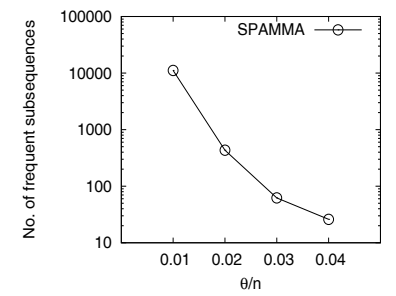

(b) No. of freq. subsequences
Fig. 6. Result for FASM in BookX by SPAMMA

Although this number is large, the modular operation over this number can be done efficiently with the GMP library [20].

Result for FASM: We conducted experiments for the FASM problem on the BookX dataset. We insert MA as an operator in SPAM [8] for FASM. Due to the bitmap representation of database, SPAM cannot process attribute-based sequences of length more than 64, which are common in Netflix and BookX. The SPAM using our operator $M A$ is denoted by SPAMMA. We compare SPAMMA with the SPAM algorithm without using our $M A$ operator, denoted by $A S P A M$. The experimental results can be found in Figure 6 where $\theta / n$ is the frequency threshold in fraction.

In Figure 6(a), the execution time of SPAMMA is much smaller than ASPAM. When $\theta$ is larger, fewer subsequences are checked whether they are frequent according to the given threshold $\theta$. So, the resulting frequent subsequence set is also smaller, as shown in Figure 6(b).

Case Study for FASM: By running SPAMMA, we found some interesting frequent subsequences. For example, when $\theta / n$ is set to 0.01 , we can find " $<$ Deutscher Taschenbuch Verlag $>,<$ Piper $>$ " as a frequent subsequence. "Deutscher Taschenbuch Verlag" is a publisher, while "Piper" is a book. Note that "Deutscher Taschenbuch Verlag" and "Piper" belong to different attributes. Another interesting case is a frequent subsequence, " $<$ She's Come Undone $>$ ". It is a book with two versions published by two different publishers: one is Washington Square Press, and the other is Pocket Books. In the result, " $<$ She's Come Undone, Washington Square Press $>$ " is also a frequent subsequence, but " $<$ She's Come Undone, Pocket Books>" is not. It means that compared with the Pocket Books version, readers prefer the Washington Square Press version.

Summary: Compressed Synopsis gives a very low compression rate. On average, the compression rate is about $25 \%$. In most cases, the execution time of MAI is an order of magnitude better than that of Naive. In the real dataset Netflix, the execution time of MAI is $3 \%$ of that of Naive.

\section{CONCLUSION}

In this paper, we propose a new problem called Attributebased Subsequence Matching Problem which has many applications. We propose an efficient algorithm for this problem using Chinese Remainder Theorem to compress each sequence into a triplet of numbers. We also illustrate how this problem can be used for mining frequent subsequences. Finally, we conducted experiments to show that our algorithm is very efficient, nearly two orders of magnitude better than the straightforward method. There are a lot of possible future directions. One direction is to consider the problem with additional constraints like gap constraints [6] commonly adopted in bioinformatics. Another direction is to study how other data mining problems about subsequence matching can be extended when the property table is considered.

Acknowledgements: The research of Yu Peng, Raymond ChiWing Wong and Liangliang Ye is supported by HKRGC GRF 621309 and Direct Allocation Grant DAG11EG05G. The research of Philip S. Yu is supported by US NSF through grants DBI-0960443, IIS 0905215, OISE-0968341 and OIA0963278, and Google Mobile 2014 Program.

\section{REFERENCES}

[1] G. Dong and J. Pei, Sequence Data Mining (Advances in Database Systems). Springer-Verlag New York, Inc., 2007.

[2] C. Faloutsos, M. Ranganathan, and Y. Manolopoulos, "Fast subsequence matching in time-series databases," in SIGMOD Rec., 1994.

[3] V. Athitsos, P. Papapetrou, M. Potamias, G. Kollios, and D. Gunopulos, "Approximate embedding-based subsequence matching of time series," in SIGMOD, 2008.

[4] W.-S. Han, J. Lee, Y.-S. Moon, and H. Jiang, "Ranked subsequence matching in time-series databases," in $V L D B, 2007$.

[5] L. Boasson, P. Cegielski, I. Guessarian, and Y. Matiyasevich, "Windowaccumulated subsequence matching problem is linear," in PODS, 1999.

[6] M. Zhang, B. Kao, D. Cheung, and K. Yip, "Mining periodic patterns with gap requirement from sequences," in SIGMOD, 2005.

[7] S. lyong Lee, S. ju Chun, D. hwan Kim, J. hong Lee, and C.-W. Chung, "Similarity search for multidimensional data sequences," in ICDE, 2000.

[8] J. Ayres, J. Flannick, J. Gehrke, and T. Yiu, "Sequential pattern mining using a bitmap representation," in KDD, 2002.

[9] R. She, F. Chen, K. Wang, M. Ester, J. L. Gardy, and F. S. L. Brinkman, "Frequent-subsequence-based prediction of outer membrane proteins," in KDD, 2003.

[10] A. Pol and T. Kahveci, "Highly scalable and accurate seeds for subsequence alignment," in BIBE: Proceedings of the Fifth IEEE Symposium on Bioinformatics and Bioengineering, 2005.

[11] G. D. Stormo, "Dna binding sites: representation and discovery." in Bioinformatics, 2000.

[12] X. Liu, D. L. Brutlag, and J. S. Liu, "Bioprospector: Discovering conserved dna motifs in upstream regulatory regions of co-expressed genes," in Pac. Symp. Biocomput, 2001.

[13] C. Wu, M. Berry, S. Shivakumar, and J. McLarty, "Neural networks for full-scale protein sequence classification: Sequence encoding with singular value decomposition," in Machine Learning, 1995.

[14] P. Geurts, A. B. Cuesta, and L. Wehenkel, "Segment and combine approach for biological sequence classification," in In: Proceedings of IEEE Symposium on Computational Intelligence in Bioinformatics and Computational Biology, 2005.

[15] T.F.Smith and M.S.Waterman, "Identification of common molecular subsequences," in Journal of Molecular Biology, 1981.

[16] S. F. Altschul, W. Gish, W. Miller, E. W. Myers, and D. J. Lipman, "Basic local alignment search tool," in Journal of molecular biology, 1990.

[17] R. T. Ng, L. V. S. Lakshmanan, J. Han, and A. Pang, "Exploratory mining and pruning optimizations of constrained associations rules," in SIGMOD Rec., 1998.

[18] J. Pei and J. Han, "Can we push more constraints into frequent pattern mining?" in $K D D, 2000$.

[19] G. H. Hardy and E. M. Wright, An Introduction to the Theory of Numbers. Oxford, England: Clarendon Press, 1979.

[20] "http://gmplib.org/."

[21] R. Srikant and R. Agrawal, "Mining sequential patterns: Generalizations and performance improvements," in EDBT, 1996.

[22] Y. Koren, "Collaborative filtering with temporal dynamics," in KDD. New York, NY, USA: ACM, 2009, pp. 447-456.

[23] "http://www.informatik.uni-freiburg.de/ cziegler/bx/."

[24] "http://www.cse.ust.hk/ raywong/genealogy/." 